\title{
Comparison of Diabetic Macular Edema Treatment Patterns and Outcomes by European and US Retina-treating Ophthalmologists
}

\author{
Eric G John, $\mathrm{MS}^{1}$ and James F Harris, $\mathrm{BBA}^{2}$
}

1. Senior Vice President, SciMedica Group, Conshohocken, Pennsylvania, US; 2. Executive Director, Marketing, Alimera Sciences, Alpharetta, Georgia, US

\begin{abstract}
AIM: To uncover and evaluate diabetic macular edema (DME) patient treatment patterns in the US and the largest EU countries. METHODS: Quantitative analyses of self-report data from retina-treating ophthalmologists via 30-minute Internet-based surveys. Data were collected across the US (one study) and EU (two studies-EU-1 and EU-2) between November 2011 and January 2013. Visual outcomes and rates of treatment success or failure were evaluated. RESULTS: Respondents were surveyed across the US ( $n=71)$ and EU (EU-1: $n=180 ; E U-2: n=106)$. Nearly one-third of treated patients (US 38.6\%; EU-1 37.8\%; EU-2 30.7 \%) reached a plateau in vision improvement. Geographical comparison showed no significant difference in responses between the US and the EU (EU-1, EU-2) ( $p>0.05)$. CONCLUSIONS: These data help to quantify the size of the chronic DME patient population in the US and EU, which is not achieving an acceptable functional outcome with current therapy. The paper provides solid empirical evidence regarding similarities in DME patient treatment and outcomes in the US and EU. The global and reproducible similarities in terms of physicians' perceptions of the threshold of DME treatment burden and the visual outcomes when treatment benefit is limited are compelling. This is especially interesting within the present context where information regarding the effect of current treatments is limited. Diabetes is growing around the world at epidemic proportions, and these data provide insight into the impact DME may have on healthcare systems.
\end{abstract}

\section{Keywords}

Diabetic macular edema, DME, treatment, epidemiology, United States, Europe

\begin{abstract}
Disclosure: Eric G John, MS, is an employee of SciMedica Group Marketing Research and Consulting. James F Harris, BBA, is an employee of Alimera Sciences. Alimera Sciences provided funding for this research. The sponsor participated in the study design and manuscript review and approval, but was not involved in the review, synthesis, and interpretation of the results.

Received: December 4, 2013 Accepted: January 30, 2014 Citation: US Ophthalmic Review, 2014;7(1):63-7 DOl: 10.17925/USOR.2014.07.01.63

Correspondence: Eric G John, MS, 20 E 2nd Ave, Conshohocken, PA 19428, US. E: ejohn@scimedicagroup.com
\end{abstract}

Support: The publication of this article was supported by Alimera Sciences. The views and opinions expressed are those of the author and not necessarily those of Alimera Sciences.

The global medical, financial, and personal toll of type 1 and 2 diabetes is well documented. In the US, the total cost of diabetes to the US healthcare system was estimated at US\$174 billion (€126 billion) in 2007. ${ }^{1}$ The International Diabetes Federation estimates that diabetes health costs were US $\$ 105.5$ billion (€82.2 billion) in the European region in $2010 .{ }^{2}$ As the prevalence of diabetes is expected to rise more than $50 \%$ globally between 2000 and 2030, these costs are expected to continue to grow. ${ }^{3}$ While robust data regarding the impact of type 1 and 2 diabetes are readily available, information on at least one condition secondary to diabetes, specifically diabetic macular edema (DME), is lacking in publically available literature. A particular gap exists regarding the relationship between the scope of the disease and the corresponding effectiveness of long-term treatment.

Highlighting this lack of information, a 2010 literature review on the burden of illness of DME in the US and Europe uncovered 47 articles pertinent to the discussion of the impact of the disease between 1980 and 2009. ${ }^{4}$
The authors concluded that little was known about the epidemiology of DME in Europe. The lack of recent data in the US and limited European epidemiological studies based on relatively small patient populations has led to a call for more research in this arena. ${ }^{4}$

Even when information regarding the burden of DME treatment is robust, it is often no longer timely. In the US, the benchmark epidemiological study of DME is the Wisconsin Epidemiological Study of Diabetic Retinopathy (WESDR). ${ }^{5}$ The WESDR, a prospective, population-based, cohort study that enrolled diabetes patients both under and over the age of 30 from 1980 to 1982 is now over 30 years old and does not reflect currently available treatment.

Some recent and relevant analysis of the prevalence of DME among those with diabetes does, however, exist. A 2012 meta-analysis of 35 studies from 1980 to 2008 estimated the prevalence for DME to be $6.81 \%$ 
Table 1: Sample Characteristics Across Studies

\begin{tabular}{|c|c|c|c|}
\hline Sample Metrics & US Study & EU Study 1 & EU Study 2 \\
\hline Sample size & 71 & 180 & 106 \\
\hline $\begin{array}{l}\text { Estimated total population of } \\
\text { retina-treating ophthalmologists/ } \\
\text { retina specialists* }\end{array}$ & 1,800 & 4,500 & 2,600 \\
\hline $\begin{array}{l}\text { Sample size as percentage of } \\
\text { total population }\end{array}$ & $3.9 \%$ & $4.0 \%$ & $3.9 \%$ \\
\hline Failed screener process & 30 & 349 & 119 \\
\hline Failed screener as $\%$ of universe & $1.7 \%$ & $7.8 \%$ & $4.5 \%$ \\
\hline Average number of patients treated/month & 568.0 & 450.0 & 500.0 \\
\hline $\begin{array}{l}\text { Average number of DME patients } \\
\text { treated/month }\end{array}$ & 116.5 & 56.5 & 60.7 \\
\hline DME patients as percentage of practice & $20.5 \%$ & $12.4 \%$ & $12.1 \%$ \\
\hline
\end{tabular}

$\overline{D M E}=$ diabetic macular edema. ${ }^{*}$ Data on file. Alimera Sciences.

(6.74-6.89) among patients with diabetes. ${ }^{6}$ Nevertheless, the authors concluded that few current estimates of prevalence worldwide are available. Details on the impact of treatments and their level of success are even more difficult to identify for the US and EU. For example, unlike in diabetes research, limited data exist regarding the impact of longer-term therapy on visual acuity (VA) levels.

This study aimed to supplement the limited information on patients with DME and their treatment by reporting survey data related to the condition in both the US and the EU. In addition to helping to reduce the gap in publically available information on DME diagnosis and treatment, these data will identify epidemiologic and treatment similarities in DME patients from both the US and EU.

\section{Research Design and Methods Study Design}

These primary research data on patients with DME collected from retinatreating ophthalmologists were gathered in three separate but similarly designed quantitative marketing research studies sponsored by Alimera Sciences over the course of approximately 2 years from November 2011 to January 2013. The studies are referred to as:

- $\quad$ US Treatment Patterns Study 1(US) -45-minute Internet-based survey completed in November 2011 with retina-treating ophthalmologists and retina specialists in the US.

- $\quad$ EU DME Treatment Patterns Study 1 (EU-1)-30-minute Internetbased survey completed in September 2012 with retina-treating ophthalmologists and retina specialists across seven EU countries, i.e. Austria (AU), France (FR), Germany (DE), Italy (IT), Portugal (PT), Spain (ES), and the UK.

- $\quad$ EU DME Treatment Patterns Study 2 (EU-2) -30-minute Internetbased survey completed in January 2013 with retina-treating ophthalmologists and retina specialists across three EU countries, i.e. France (FR), Germany (DE), and the UK. For the EU-2 study, only the three most populous nations in Europe (France, Germany, and the UK) were included to maximize project timing and resources.

For each study, an independent panel of retina-treating ophthalmologists was recruited to participate in the survey via phone, fax, and e-mail invitation. Utilization of the panel for identification of eligible physician respondents was the most timely and cost-effective approach to assure qualified participation by appropriate retina-treating ophthalmologists.

Given the relatively small number of these specialist physicians in each country, sample sizes were determined by estimating the maximum number of physicians that could be recruited through the panel to complete a 30-minute survey within a 3-week period. Robust sample sizes ( $4 \%$ of the total population) were obtained in order to allow for a margin of error less than \pm 12 points when assessing any results relative to the total population (see Table 1). To underscore the robustness of the physician sample sizes in these studies, the average sample size for public opinion polls in the US is 1,000 respondents for a population of 200 million voting age adults. ${ }^{7}$ To further illustrate what designates an adequate sample size in this treatment area, it should be noted that the sample size for each arm of a phase III trial in DME that recently lead to a US Food and Drug Administration (FDA) product approval was 125 patients. $^{8}$

Following standard protocol for healthcare primary research, respondent identities were not revealed to the sponsor of the study during the recruiting process. Also following standard protocol, respondents were provided with honoraria for their participation through the third party panel companies who contacted them to participate in the research study. Respondents could designate donation of these honoraria to charity if that was their preference. Diversity of sample was sought on many levels in terms of geography and practice size. To reflect group practice, more than one respondent from the same practice were allowed into the study. Each physician from the practice was instructed to answer questions in the survey only about the patients he or she personally treated, and not for the practice as a whole.

The EU-1 study was structured so that seven countries representing $67 \%$ of the population in the $\mathrm{EU}^{9}$ were included. The allocation of respondents by country in the EU study was distributed based on size of the general population in each nation. As mentioned earlier, the EU-2 study included the largest nations in Europe only (France, Germany, and the UK) to maximize project timing and resources.

The results presented here are all based on self-report by the retinatreating ophthalmologists. While this approach can include inherent bias on the part of the respondent, the representative sample sizes across each study and the consistency in response to each question should significantly reduce the limitations of self-reported data. As identified in other studies, self-report procedures can provide useful estimates of consumption in clinical settings when conditions are designed to maximize response accuracy. ${ }^{10}$

\section{Screening Process and Survey Execution}

All potential physician respondents completed an extensive screening process before becoming eligible to complete the online survey. Inclusion criteria included confirmation of specialization in treating diseases of the retina, as well as the following parameters:

- Total size of practice $>99$ patients per month ( $>74$ in Austria and Portugal in EU-1).

- $\quad$ Total size of DME patient practice >nine patients per month (>19 in US).

- At least one anti-vascular endothelial growth factor (anti-VEGF) intravitreal injection for DME per month (>6 in US; 0 in UK). 
The survey used in each of the studies began with background questions identifying the profile or composition of each respondent's practice in terms of patients with DME. These questions formed the basis for comparison of similarities between the US and EU samples and included the following:

- What percentage of the DME patients you treat in a typical month are new versus existing? (Definitions of 'new' and 'existing' provided.)

- What percentage of the patients you currently treat for DME receive the following therapies?

- What percentage of DME patients typically achieve the following best-corrected VA (BCVA) levels long term?

- $\quad$ To the best of your ability, please estimate the average monthly percentage of your DME patients who fall into the 'plateaued/ insufficiently responsive patient' category. This patient type was defined as: "those that have stable optical coherence tomography [OCT] with some fluid, with vision worse than 20/40 (0.5, UK 6/12) after being treated for a period of time; essentially, these patients have reached a plateau and are 'stuck,' and the vision is no longer improving."

The list of therapies across that respondents allocated their newly diagnosed and existing patients included pharmacologic agents available at the time of the survey (bevacizumab, ranibizumab, pegaptanib, dexamethasone, triamcinolone), laser photocoagulation, and vitrectomy or a combination (pharmacologic agent with laser treatment). New patients were defined as those who had been recently diagnosed (within the last 6 months) with DME, while existing patients were those who received a diagnosis more than 6 months prior to the survey.

BCVA levels were provided with multiple conversions for every respondent so that they could answer relevant questions with the measure (Snellen, meter, decimal) most commonly used in his or her particular country (see Table 2).

Additional survey questions were either therapy- or product-specific and were not relevant to similarities in patient profiles.

\section{Statistical Analysis}

Data for each study was checked for consistency in responses. Confirmation of the absence of difference between the results for the US study and the EU-1 and EU-2 studies was completed using a test of proportions for two independent groups with a z-test under a pooled or separate variance assumption for specified proportions and sample sizes. Statistical significance was considered for $p<0.05$. For comparisons by country, sample sizes consisting of fewer than 20 respondents were considered too small for meaningful comparison. Data analysis and calculations were conducted using Statistical Analysis System (SAS ${ }^{\circledR}$ ) 9.3 (SAS, Cary, North Carolina, US).

\section{Results}

Retina-treating ophthalmologists and retina specialists were surveyed across the US (US: $n=71$ ) and Europe (EU-1: $n=180 ; A T$ [10], FR [30], DE [35], IT [30], PT [10], ES [30], UK [35]) (EU-2: $n=105 ;$ FR [35], DE [35], UK [35]). As a result of the screening exercises in the survey, 30 retina specialists did not complete the questionnaire due to failure to meet eligibility criteria in the US study (1.7\% of the total population). In
Table 2: Visual Acuity Conversions for Global Studies

\begin{tabular}{|c|c|c|}
\hline \multicolumn{3}{|c|}{ Visual Acuity Conversions } \\
\hline Snellen Feet & Meter Equivalent & Decimal \\
\hline $20 / 20$ & $6 / 6$ & 1.00 \\
\hline $20 / 40$ & $6 / 12$ & 0.50 \\
\hline $20 / 50$ & $6 / 15$ & 0.40 \\
\hline $20 / 60$ & $6 / 18$ & 0.33 \\
\hline $20 / 80$ & $6 / 24$ & 0.25 \\
\hline $20 / 100$ & $6 / 30$ & 0.20 \\
\hline
\end{tabular}

Table 3 (A and B): Comparison of Therapy Use for Patients with Diabetic Macular Edema-US and EU

\begin{tabular}{|c|c|c|c|}
\hline \multicolumn{4}{|l|}{ A. New Patients } \\
\hline Therapies Used & $\begin{array}{l}\text { US Study } 2 \\
(n=71)\end{array}$ & $\begin{array}{l}\text { EU Study } 1 \\
(n=180)\end{array}$ & $\begin{array}{l}\text { EU Study } 2 \\
(n=106)\end{array}$ \\
\hline Anti-VEGF with or & $59.2 \%$ & $57.1 \%$ & $57.8 \%$ \\
\hline without laser & $(47.5-70.0 \%)$ & (49.8-64.1\%) & $(48.2-66.8 \%)$ \\
\hline Steroids with or & $10.7 \%$ & $14.9 \%$ & $14.1 \%$ \\
\hline without laser & $(5.3-20.3 \%)$ & (10.4-20.9\%) & $(8.7-22.1 \%)$ \\
\hline \multirow[t]{2}{*}{ Laser alone } & $26.9 \%$ & $22.6 \%$ & $26.3 \%$ \\
\hline & (17.9-38.3\%) & (17.1-29.3\%) & (18.8-35.5 \%) \\
\hline \multirow[t]{2}{*}{ Other } & $3.2 \%$ & $5.5 \%$ & $1.7 \%$ \\
\hline & $(0.9-11.0 \%)$ & (3.0-10.0 \%) & $(0.4-7.0 \%)$ \\
\hline \multicolumn{4}{|c|}{ B. Existing Patients } \\
\hline Therapies Used & $\begin{array}{l}\text { US Study } 2 \\
(n=71)\end{array}$ & $\begin{array}{l}\text { EU Study } 1 \\
(n=180)\end{array}$ & $\begin{array}{l}\text { EU Study } 2 \\
(n=106)\end{array}$ \\
\hline \multirow{2}{*}{$\begin{array}{l}\text { Anti-VEGF with or } \\
\text { without laser }\end{array}$} & $57.4 \%$ & $58.1 \%$ & $59.2 \%$ \\
\hline & (45.7-68.3\%) & (50.8-65.1\%) & (49.6-68.1\%) \\
\hline \multirow{2}{*}{$\begin{array}{l}\text { Steroids with or } \\
\text { without laser }\end{array}$} & $17.7 \%$ & $17.6 \%$ & $16.8 \%$ \\
\hline & $(10.5-28.4 \%)$ & (12.7-23.9\%) & $(10.8-25.2 \%)$ \\
\hline \multirow[t]{2}{*}{ Laser alone } & $20.4 \%$ & $18.5 \%$ & $22.0 \%$ \\
\hline & $(12.6-31.3 \%)$ & (13.5-24.9\%) & (15.1-30.9\%) \\
\hline \multirow[t]{2}{*}{ Other } & $4.4 \%$ & $5.9 \%$ & $1.8 \%$ \\
\hline & $(1.5-12.5 \%)$ & (3.3-10.4 \%) & $(0.5-7.1 \%)$ \\
\hline
\end{tabular}

Anti-VEGF $=$ anti-vascular endothelial growth factor.

the EU-1 study, a greater number of potential respondents failed the screening process (349 or $7.8 \%$ of the total population) thus helping to ensure that the final sample was representative of DME-treating physicians (see Table 1). The mean number of all patients seen in US practices was 568 (US-2). Physicians in the EU-1 study each maintained a mean of 450 patients in their practices, while those in the EU-2 study saw 500 patients on average. The mean proportion of patients with DME in each respondent's practice was $20.5 \%$ and $12.4 \%$ and $12.1 \%$ for the US, EU-1, and EU-2 samples, respectively.

\section{Outcomes for Patients with Diabetic Macular Edema}

Similarities were apparent between the US and the EU in terms of the types of therapies used to treat DME. Table 3 illustrates the estimated use, by class of therapy, for new and existing patients with DME in both the US (US) and EU (EU-1; EU-2). In terms of therapeutic approaches, the mean use of anti-VEGF therapies was $59.2 \%, 57.1 \%$, and $57.8 \%$ for new patients in the US and EU (EU-1; EU-2), respectively (see Table 3A). For existing patients, mean use was $57.4 \%$ in the US, while in the EU it was $58.1 \%$ and $59.2 \%$, respectively (B). Laser monotherapy ranked second in 
Table 4. Estimated Visual Acuity Levels Post-therapy by Location

\begin{tabular}{|c|c|c|c|c|}
\hline Visual Acuity Achievement Levels & US Study $2(n=71)$ & EU Study $1(n=180)$ & EU Study $2(n=106)$ & EU Study 1 and $2(n=286)$ \\
\hline a. $B C V A<20 / 40(0.50)(U K=6 / 12)$ & $44.8 \%$ (33.5-56.1\%) & $43.8 \%(36.6-60.0 \%)$ & 41.3 \% (32.3-50.9\%) & 42.9 \% (37.2-48.5 \%) \\
\hline b. BCVA 20/60 (0.33) (UK=6/18) & $22.7 \%(13.2-32.3 \%)$ & $24.3 \%(18.1-30.5 \%)$ & $25.3 \%$ (17.9-34.4 \%) & $24.7 \%$ (19.8-29.7 \%) \\
\hline c. BCVA >20/80 (0.25) $(U K=6 / 24)$ & $32.5 \%$ (21.8-43.2 \%) & $31.9 \%$ (25.2-38.7 \%) & $33.4 \%$ (25.1-42.9\%) & $32.4 \%$ (27.1-37.8 \%) \\
\hline Sum of $b+c>20 / 60(0.33)(U K=6 / 18)$ & $55.2 \%(43.6-66.3 \%)$ & $56.2 \%$ (48.9-63.3 \%) & $58.7 \%$ (49.1-67.7 \%) & $57.1 \%(51.5-62.8 \%)$ \\
\hline
\end{tabular}

$B C V A=$ best-corrected visual acuity.

Table 5: Patient Characteristics by Country

\begin{tabular}{llll} 
US Study & $\begin{array}{l}\text { EU Study 1 } \\
(n=71)\end{array}$ & $\begin{array}{l}\text { EU Study 2 } \\
(n=106)\end{array}$ \\
Patient Type & $24.2 \%$ & $32.2 \%$ & $30.4 \%$ \\
New diabetic macular & $(14.4-34.0 \%)$ & $(25.4-39.9 \%)$ & $(24.1-39.8 \%)$ \\
edema patients & $75.8 \%$ & $67.8 \%$ & $69.6 \%$ \\
Existing diabetic macular & $(66.0-85.6 \%)$ & $(61.0-74.6 \%)$ & $(60.2-77.6 \%)$ \\
edema patients & & & \\
\hline
\end{tabular}

terms of the physicians' reported utilization. All other treatments, such as steroids and vitrectomy, were used in $<20 \%$ of patients with DME.

Data obtained from the US and EU retina-treating ophthalmologists included an assessment of the degree to which patients' VA did or did not improve with therapy over time. After 2 or more years of therapy, respondents reported achieving BCVA $<20 / 40$ in two of five of their patients with DME (US $44.8 \%$; EU-1 $43.8 \%$; EU-2 41.3 \%) (see Table 4). Another one in five patients (US 22.7 \%; EU-1 $24.3 \%$; EU-2 $25.3 \%$ ) had vision impairment measuring 20/60 after therapy. One in three patients with DME (US 32.5 \%; EU-1 $31.9 \%$; EU-2 33.4 \%) continued to experience even greater vision impairment with BCVA of $20 / 80$ or worse.

On a separate but related question, respondents were asked to estimate the percentage of their patients with DME who were insufficiently responsive or who had reached a plateau in treatment response. These patients were defined in the survey as, "those who have stable optical coherence tomography (OCT) scans with some fluid, with vision worse than 20/40 while on treatment; essentially, these patients are 'stuck' and the vision is no longer improving." Physicians in the US estimated that $38.6 \%$ of their patients with DME met these criteria, while in the EU the estimate was $37.8 \%$ in EU-1 and $30.7 \%$ in EU-2. This result averaged $35.2 \%$ across the combined two waves in the EU.

\section{Patient Characteristics}

Comparison of the US and EU samples revealed no statistically significant difference $(p>0.05)$ in patient characteristics between physician responses. Patients with DME identified as 'new' (US 24.2 \%; EU-1 $32.2 \%$; EU-2 30.4 \%) or 'existing' (US 75.8 \%; EU-1 67.8 \%; EU-2 $69.6 \%$ ) differed by $<10$ percentage points between the US and EU samples (see Table 5).

\section{Conclusions}

The purpose of reporting the study results is to identify the size and characteristics of clinically important subgroups of patients with DME, in order to estimate the functional outcomes of these subgroups over time. These data provide new and valuable insight into the DME patient population in the US and EU given the previously identified gaps in timely, publically available patient information. No other publically available data regarding the recent levels of therapy allocated across patients with DME exists in publication in the US or EU.

The size of the samples for each region and the reproducibility illustrated suggest that the results are not isolated anomalies and may provide a useful picture of patient metrics and therapy usage for DME. The screening criteria achieved the goal of ensuring respondents to the surveys were representative of the US and EU population of physicians who most often treat DME patients. The similarities among the US and EU retina-treating ophthalmologists surveyed are also not surprising given the existence of internationally tested and approved approaches to treat DME such as anti-VEGF therapy.

With regard to anti-VEGF therapy, the most common treatment approach in the research studies, the data revealed that utilization was $>50 \%$ for new and existing patients. Our research suggests that available treatment algorithms clearly benefit a segment of the patient population with DME, specifically the $40 \%$ or more whose vision improves to $20 / 40$ or better with therapy. However, $22.7 \%$ (US) to $25.3 \%$ (EU-2) of treated patients with DME never improved above a BCVA of 20/60. Another third of patients (US $32.5 \%$; EU-1 $31.9 \%$; EU-2 33.4 \%) never improved above a BCVA of 20/80 (55.2\% US to $58.7 \%$ EU-2 combined) (see Table 4). An outcome of at least some functional vision impairment (BCVA 20/60 or worse) for $50 \%$ or more of patients with DME suggests the existence of a subgroup with chronic DME who may benefit from new or alternative treatment options.

Maximizing VA or vision improvement in the patient population with DME is critical to managing the societal impact of the disease. In the US, $93.6 \%$ of individuals aged 12 years or older have VA of $20 / 40$ or better in their betterseeing eye. ${ }^{11}$ Furthermore, only $8.8 \%$ of patients age 60 or older have visual impairment defined as 20/50 vision or worse in the better-seeing eye with visual correction. ${ }^{11}$ The levels of VA required to drive vary by state but generally range from $20 / 40$ to $20 / 60$. In the UK, corrected vision of $6 / 12$ (20/40) or better is required to drive. ${ }^{12,13}$ Thus, VA worse than 20/40 appears to be a consensus determination for visual impairment. The results of our research indicate that a sizable portion of the treated patient population falls outside the visual requirements for activities such as driving a vehicle.

Our research suggests that a relatively high ratio of patients with DME have been diagnosed in the past 6 months (US $23.7 \%$; EU-1 $32.2 \%$; EU-2 $30.4 \%$ ). This represents a challenge to retina-treating ophthalmologists, as these patients may utilize more of the resources of a practice due to the likelihood of chronic disease or suboptimal visual outcomes, which appear to occur in up to half of cases. This patient demographic coupled with the need for frequent patient interactions in order to maximize antiVEGF therapy will only increase the burden on ophthalmology services. And while no direct comparison can be made, recent data from several 
phase III clinical trial programs ${ }^{8,14}$ seem to suggest better outcomes for anti-VEGF therapy with more frequent injections.

Overall our results categorized the current DME patient population in a clinically relevant manner in terms of type and course of therapy as well as patients' response to that therapy. In addition, they revealed the existence of a subgroup experiencing a 'plateau' in terms of functional response based on the currently available therapies, and identified both vision and treatment burdens. We hope the further clarification of the needs of those with DME will help to drive the optimization of specialized services and treatment paradigms as well as the development of new therapeutic agents in the future.
1. American Diabetes Association. Diabetes Statistics, January 2011. Available at: http://www diabetes org/diabetes-basics/ diabetes-statistics/?loc=DropDownDB-stats (accessed November 27, 2012)

2. World Health Organization. Diabetes epidemic in Europe. Available at: http://www.euro.who.int/en/what-we-do/ health-topics/noncommunicable-diseases/diabetes/news/ news/2011/11/diabetes-epidemic-in-europe (accessed November 27, 2012)

3. Wild S, Roglic G, Green A et al., Global prevalence of diabetes: estimates for the year 2000 and projections for 2030, Diabetes Care, 2004;27:1047-53.

4. Chen $E$, Looman M, Laouri M, et al., Burden of illness of DME: literature review, Curr Med Res Opin, 2010;26:1587-97.

5. Klein R, Klein B, Moss S, Epidemiology of proliferative diabetic retinopathy, Diabetes Care, 1992:15:1875-91.
6. Yau J, Rogers S, Kawasaki R, et al., Meta-analysis for eye disease (META-EYE) study group. Global prevalence and major risk factors of diabetic retinopathy, Diabetes Care 2012;35:556-64.

7. National Council on Public Polls. FAQS Answers to Questions we Often Hear from the Public. Available at: http://www.ncpp. org/?q=node/6 (accessed January 7, 2013).

8. Nguyen Q, Brown D, Marcus D, et al., Ranibizumab for diabetic macular edema. Results from 2 Phase III randomized trials: RISE and RIDE, Ophthalmol, 2012:119:789-801.

9. Eurostat, Population at 1 January 2001-2012. Available at: http://epp.eurostat.ec.europa.eu/tgm/table.do?tab=tab le\&language $=$ en $\&$ pcode $=$ tps00001\&tableselection $=1 \&$ foo notes $=$ yes\&labeling=labels\&plugin $=1$ (accessed November 27, 2012)

10. Del Boca FK, Noll JA, Truth or consequences: the validity of self-report data in health services research on addictions, Addiction, 2000:\$3:47-60.

11. Vitale $S$, Cotch M, Sperduto R, Prevalence of visual

impairment in the United States, JAMA, 2006;295:2158-63.

12. Driver and Vehicle Licensing Agency. A guide to standards of vision for driving cars and motorcycles (Group 1),

July 2012. Available at: http://www.direct.gov.uk/prod_ consum_dg/groups/dg_digitalassets/@dg/@en/@motor/ documents/digitalasset/dg_065298.pdf (accessed November 27. 2012).

13. Hirani S, Vision standards for driving - the evidence base Optometry Today, 2001;51:37-9.

14. Mitchell P, Bandello F, Schmidt-Erfurth $U$, et al. The RESTORE study: ranibizumab monotherapy or combined with laser versus laser monotherapy for diabetic macular edema, Ophthalmol, 2001:118:615-25. 